\title{
Myosin II motor activity in the lateral amygdala is required for fear memory consolidation
}

\author{
Cristin F. Gavin, ${ }^{1,2}$ Maria D. Rubio, ${ }^{3}$ Erica Young, ${ }^{1,4}$ Courtney Miller, ${ }^{1,4}$ \\ and Gavin Rumbaugh ${ }^{1,5}$
}

\begin{abstract}
${ }^{1}$ Department of Neuroscience, The Scripps Research Institute, Jupiter, Florida 33458, USA; ${ }^{2}$ Neuroscience Graduate Program, The University of Alabama at Birmingham, Birmingham, Alabama 35294, USA; ${ }^{3}$ Department of Psychiatry, The University of Alabama at Birmingham, Birmingham, Alabama 35294, USA; ${ }^{4}$ Department of Metabolism and Aging, The Scripps Research Institute, Jupiter, Florida 33458, USA
\end{abstract}

\begin{abstract}
Learning induces dynamic changes to the actin cytoskeleton that are required to support memory formation. However, the molecular mechanisms that mediate filamentous actin (F-actin) dynamics during learning and memory are poorly understood. Myosin II motors are highly expressed in actin-rich growth structures including dendritic spines, and we have recently shown that these molecular machines mobilize F-actin in response to synaptic stimulation and learning in the hippocampus. In this study, we report that Myosin II motors in the rat lateral amygdala (LA) are essential for fear memory formation. Pretraining infusions of the Myosin II inhibitor, blebbistatin (blebb), disrupted long term memory, while short term memory was unaffected. Interestingly, both post-training and pretesting infusions had no effect on memory formation, indicating that Myosin II motors operate during or shortly after learning to promote memory consolidation. These data support the idea that Myosin II motor-force generation is a general mechanism that supports memory consolidation in the mammalian CNS.
\end{abstract}

[Supplemental material is available for this article.]

Long-term memory (LTM) formation is traditionally considered to begin with the acquisition of a novel association followed by multiple protein synthesis-dependent consolidation mechanisms (Dudai 2002; Rodrigues et al. 2004; Morris 2006). It has been hypothesized that memory consolidation proceeds slowly in order to provide an extended window in which recently acquired information can be stored, modulated, or erased. The existence of an early phase of memory formation has been proposed to account for the apparent lability of associative memories shortly after learning (McGaugh 2000; Dudai 2004). Furthermore, there is evidence for the activation of parallel mechanisms during learning to keep a memory active until longer-term modifications are completed (McGaugh 2000; Izquierdo et al. 2002). Despite the importance of these early memory processes, their underlying mechanisms are virtually unknown (Dudai 2004).

Synaptic plasticity is an excellent candidate for an early consolidation mechanism because it occurs at the time of associative learning in several brain regions (Rogan et al. 1997; Malenka and Bear 2004; Whitlock et al. 2006) including the lateral amygdala (LA) (Rogan et al. 1997). Structural and functional modifications of synapses rely on activity-dependent remodeling of the actin cytoskeleton (Lamprecht and LeDoux 2004; Dillon and Goda 2005; Hanley 2008). Importantly, this process is integral to LTM formation across various brain regions including those regions essential for associative learning (Fischer et al. 2004; Mantzur et al. 2009). Thus, understanding the molecular mechanisms that participate in the dynamic reorganization of the actin cytoskeleton will likely contribute to our understanding of how memories form.

\footnotetext{
${ }^{5}$ Corresponding author.

E-mail grumbaug@scripps.edu.

Article is online at http://www.learnmem.org/cgi/doi/10.1101/lm.024042.111.
}

Nonmuscle Myosin II, a hexameric protein complex found in all cell types, is a molecular motor that dramatically regulates actin dynamics in growth structures (Lin et al. 1996; Brown and Bridgman 2004; Medeiros et al. 2006). Recently, our group reported that theta stimulation activates synaptic Myosin II motor force generation in the hippocampus which, in turn, promotes spine F-actin polymerization (Rex et al. 2010). This process reorganizes the cytoskeleton at synapses and is required for the stable expression of both long-term potentiation (LTP) and long-term contextual memory. In addition, we and others have found a rich diversity of functional Myosin II isoforms in dendritic spines (Brown and Bridgman 2003; Ryu et al. 2006; Rubio et al. 2011). While these studies have demonstrated the importance of Myosin II motors in the hippocampus, it is unclear if Myosin II motors are required for plasticity and memory in other brain regions.

Here we report that Myosin II ATPase activity in the LA is required for a very early phase of LTM consolidation. We identified Myosin IIB as an essential motor isoform required for amygdala-dependent associative memory formation. These data indicate that Myosin IIB motor activity is a conserved process underlying LTM formation.

\section{Results}

Pavlovian fear conditioning is a valuable behavioral paradigm used to study the neural foundations of emotional learning and memory. In this paradigm, an association is formed between a neutral conditioned stimulus (CS; auditory tone) and an aversive unconditioned stimulus (US; foot shock). Following training, the CS elicits a predictable conditioned response (CR) that serves as a measure of memory created during learning (Maren 2001; Rodrigues et al. 2004). To specifically measure a cued fear response, it is essential to test animals in a context distinct from the training 
environment. Thus, the difference in freezing between pretone and tone portions of the retrieval test is a reflection of the cueshock association, which is an accepted proxy of memory strength.

To confirm the validity of our behavioral assay, adult rats were trained in a novel context using either a paired associative learning paradigm where the CS and US were presented contemporaneously, or a control nonassociative learning paradigm, where the CS and US were presented separately. Testing was carried out in a second context $24 \mathrm{~h}$ later, where the animals were presented with the tone and their freezing behavior was quantified as an indicator of fear memory for the tone. Animals conditioned with the paired protocol demonstrated significantly more freezing to the tone relative to the pretone period (Fig. 1), indicating that our training and testing contexts are sufficiently distinct to elicit a pure tone-dependent memory. Importantly, rats trained with the unpaired protocol did not exhibit elevated freezing levels (Fig. 1). Finally, rats trained with the paired protocol demonstrated significantly more freezing to the tone compared to rats trained with the unpaired protocol (Fig. 1), indicating that tone-dependent freezing elicited after training using this protocol is purely associative in nature. Thus, the difference between pretone and tone freezing during an expression test is reflective of the amygdaladependent cued fear memory, which negates the need to perform unpaired controls in subsequent experiments.

A large body of experimental evidence indicates that the LA is the site of acquisition and retention of fear conditioning when a discrete stimulus, such as an auditory tone, is used (LeDoux 2000; Blair et al. 2001; Maren 2001; Schafe et al. 2001; Rodrigues et al. 2004). Long-lasting plasticity at LA synapses is seen following pairing of the CS and US during cued fear conditioning (Rogan et al. 1997). Thus, in order to test the idea that Myosin II activity is necessary for long-term fear memory formation, we infused the Myosin II ATPase inhibitor, blebbistatin (blebb), directly into the LA through bilateral cannulae. Blebb is a potent and specific inhibitor of Myosin II motors, which acts by inhibiting the ATP hydrolase activity of the Myosin II head (Kovacs et al. 2004; Limouze et al. 2004; Allingham et al. 2005; Rex et al. 2010). Animals were given bilateral intra-LA infusions of blebb or the inactive enantiomer (denoted "active" and "inactive," respectively) $30 \mathrm{~min}$ prior to cued fear training and then assessed for fear memory $24 \mathrm{~h}$ later (Fig. 2A). Animals infused with blebb displayed less freezing behavior than controls, indicating that inhibition of Myosin II ATPase activity in the LA prevents long-term fear memory formation (Fig. 2B). Following behavioral testing,

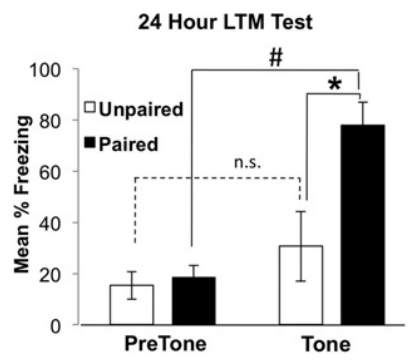

Figure 1. Validation of the cued fear conditioning experimental paradigm. Cued fear conditioning of Sprague-Dawley rats, no cannulations. Paired animals froze more during the tone presentation than unpaired animals in a distinct context $\left(F_{(1,9)}=8.39\right.$, paired $n=5$, unpaired $n=5$; * denotes significant difference between paired and unpaired animals, $P<0.02)$. Differences between pretone and tone freezing demonstrate that freezing is tone-dependent in the paired group only $\left(F_{(1,4)}=34.10\right.$, $P<0.001$; \# denotes significant difference between pretone and tonedependent freezing in the paired group; unpaired: $F_{(1,4)}=1.10, P>$ 0.05). Error bars represent SEM.
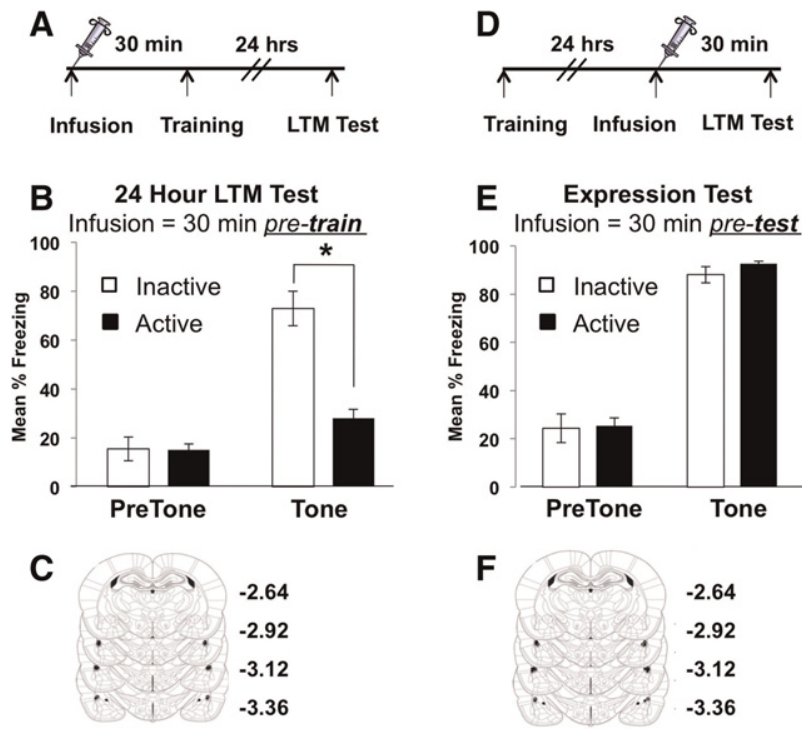

Figure 2. Inhibition of Myosin II ATPase activity in the lateral amygdala blocks the formation, but not expression, of long-term fear memory. $(A)$ Schematic of experimental design for LTM test. (B) Intra-LA infusions of the Myosin II ATPase inhibitor, blebb, prior to cued fear conditioning blocks memory formation $\left(F_{(1,10)}=26.90 ; n=5\right.$, inactive; $n=6$, active blebb; * denotes significantly different LTM at $24 \mathrm{~h}, P \leq 0.001)$. (C) Location of needle tips for all intra-LA infusions. Diagram represents histology from animals whose behavioral data are depicted in $B$. (D) Schematic of experimental design for memory expression test. $(E)$ Infusions of blebb into the LA 30 min prior to a LTM test have no effect on memory expression $\left(F_{(1,10)}=0.15, P>0.05 ; n=5\right.$, inactive; $n=6$, active blebb). $(F)$ Location of needle tips for all intra-LA infusions given to animals whose behavioral data are depicted in $E$. Because of the extensive overlap of the infusion needle tips between animals, not all tip locations are resolvable on the diagrams. Error bars represent SEM.

all animals were euthanized, and the cannulae infusion tips were confirmed for localization in the LA (Fig. 2C; Supplemental Fig. S1). A concern when infusing a small molecule inhibitor into the brain is that it may disrupt neuronal function in a nonspecific way, which would obviously impact memory encoding. However, it has been previously shown that the same neurons in the LA are needed to both encode and express a fear memory (Reijmers et al. 2007; Han et al. 2009; Zhou et al. 2009). Thus, pretest infusions should have no effect on the already formed and stabilized memory if it is, indeed, specific to an effect on Myosin II function occurring at the time of learning. To confirm this, we infused blebb $30 \mathrm{~min}$ prior to testing (Fig. 2D). There was no effect of blebb on memory expression (Fig. 2E), indicating that blebb infusions do not compromise neuronal firing or the molecular mechanism of fear memory retrieval. Infuser locations were confirmed following the experiment, and all infusions were targeted to the LA (Fig. 2F). Together, these data suggest that Myosin II plays an essential role in fear memory formation.

We next sought to determine if blebb disrupts either memory acquisition or consolidation. First, we tested the idea that inhibiting Myosin II ATPase activity during the traditional memory consolidation window would disrupt LTM formation. To do this, animals were given intra-LA infusions of active or inactive blebb 30 min after training (Fig. 3A). Interestingly, both groups displayed equally strong freezing during the 24-h expression test (Fig. 3B), suggesting that Myosin II motors may be required for memory acquisition. However, short-term memory (STM) testing (e.g., 90 min post-training) can definitively determine acquisition vs. consolidation mechanisms (Rodrigues et al. 2004). Thus, we 

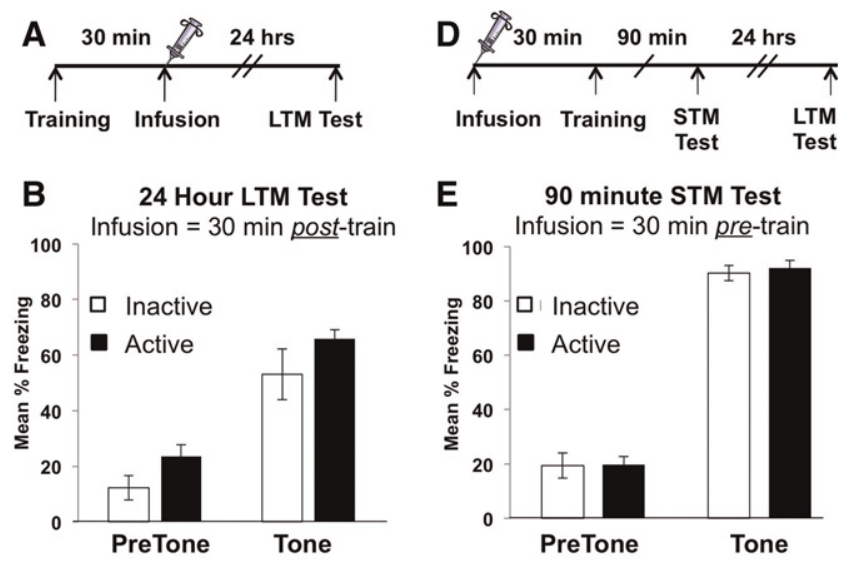

C

F

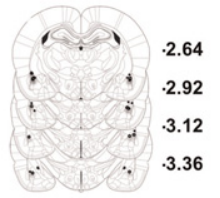

Figure 3. Myosin II motor activity is required for consolidation, not acquisition, of fear memory. (A) Schematic of experimental design for post-training blebb infusions. (B) Intra-LA infusions of the Myosin II ATPase inhibitor, blebb, $30 \mathrm{~min}$ after cued fear conditioning has no effect on fear memory formation $\left(F_{(1,14)}=0.18, P>0.05 ; n=8\right.$, inactive; $n=7$, active blebb). (C) Location of needle tips for all intra-LA infusions from animals whose behavioral data are depicted in $B$. (D) Schematic of experimental design to test the role of Myosin II in memory acquisition. (E) Pretraining intra-LA infusions of the Myosin II ATPase inhibitor blebb have no effect on STM $\left(F_{(1,11)}=0.004, P>0.05 ; n=6\right.$, inactive; $n=6$, active blebb). $(F)$ Location of needle tips for infusions depicted in $E$. Error bars represent SEM.

administered infusions of active or inactive blebb $30 \mathrm{~min}$ before training and assessed memory acquisition by quantifying freezing during training, as well as a STM test 90 min later (Supplemental Fig. S2; Fig. 3D, respectively). Surprisingly, blebb had no effect on short-term memory (Fig. 3E), demonstrating that memories are formed normally in the presence of the inhibitor but then fail to consolidate. Upon conclusion of behavioral testing, all animals were euthanized and cannulae placement was confirmed (Fig. 3F).

Our group has recently shown that the B isoform of Myosin II is crucial for both the maintenance of LTP at CA1 synapses and the consolidation of long-term contextual fear memory (Rex et al. 2010). Based on our pharmacological studies in the amygdala, combined with our previous results in the hippocampus, we hypothesized that Myosin IIB motors are required in the LA for the consolidation of LTM. To test this idea, we gave bilateral intra-LA injections of recombinant adeno-associated virus (rAAV) particles expressing a genetically encoded short hairpin RNA (shRNA) targeting Myosin IIB or a nontargeting control shRNA (NTC) (Rex et al. 2010). Following surgery, animals were returned to their home cage for 1 mo to allow the virus to reach peak expression (Fig. 4A; Rex et al. 2010). After appropriate handling and habituation, animals were fear conditioned. To assess LTM formation, freezing behavior was evaluated $24 \mathrm{~h}$ later. Animals injected with the shRNAs targeting Myosin IIB show LTM impairment, compared to animals injected with the NTC shRNAs (Fig. $4 \mathrm{~B})$. Furthermore, freezing to the CS during training was not different between groups, indicating that Myosin IIB shRNAs do not generally disrupt the freezing response (Fig. 4C). To confirm that Myosin IIB shRNAs reduced expression of this motor in the
LA, we microdissected the LA from a subset of subjects and then measured Myosin IIB protein expression by Western blot (Fig. $4 \mathrm{D})$. Animals that received the $\mathrm{AAAV}$ expressing a hairpin against Myosin IIB exhibited $\sim 50 \%$ of the expression of Myosin IIB relative to animals injected with NTC shRNAs (Fig. 4E and F). Importantly, these data also strengthen our blebb experiments (Fig. 2B) because reducing expression of a Myosin II isoform and inhibiting Myosin II motor activity in the LA have similar effects on fear memory. Taken together, these data demonstrate that Myosin II motor expression and function is necessary for the formation of long-term amygdala-dependent fear memory.

Actin polymerization in both CA1 hippocampus (Fischer et al. 2004; Rex et al. 2010) and the lateral amygdala (Lamprecht et al. 2006; Mantzur et al. 2009) is required for memory formation. Recently, we demonstrated that Myosin IIB motor activity is needed for activity-induced increases in F-actin polymerization in CA1 postsynapses, which were also required for memory formation (Rex et al. 2010). Therefore, we hypothesized that Myosin II motor activity and F-actin polymerization participate in a similar molecular process that underlies memory consolidation in the LA. First, we confirmed the results from a previous study (Mantzur et al. 2009) demonstrating that preventing actin polymerization in the LA disrupts memory formation (Fig. 5A-C). Bilateral intraamygdala infusions of latrunculin (LatA), or vehicle, were given 15 min prior to cued fear conditioning (Fig. 5A) to prevent actin polymerization. Tone-induced freezing was assessed $24 \mathrm{~h}$ later.

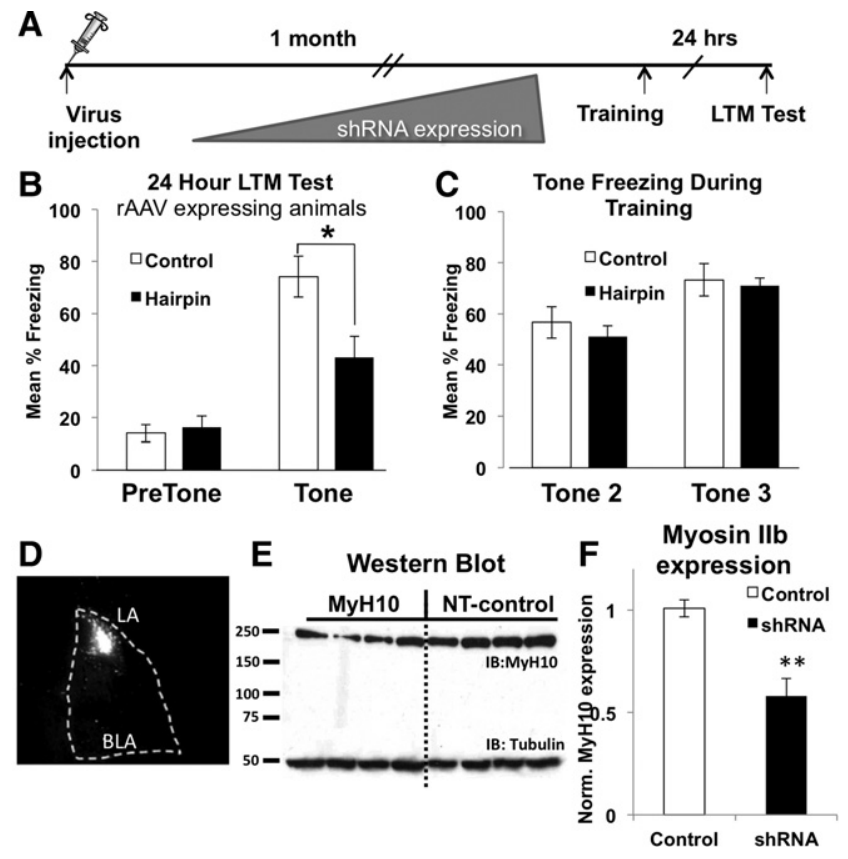

Figure 4. Reducing Myosin IIB heavy chain expression in the lateral amygdala disrupts fear memory formation. $(A)$ Schematic of experimental design for in vivo disruption of Myosin IIB. (B) In vivo knockdown of Myosin IIB disrupts fear memory formation $\left(F_{(1,15)}=7.32, P<0.05\right.$; $n=7$, NTC shRNA; $n=9$, MYH10 hairpin; * denotes significantly different LTM at $24 \mathrm{~h}$ ). (C) In vivo reduction of Myosin IIB does not affect amygdala-dependent associative learning or the ability to freeze $\left(F_{(2,15)}=0.37, P>0.05\right)$. (D) Representative image of a brain section from an animal injected with rAAV NTC-GFP. Dashed line outlines the lateral and basolateral amygdala. GFP expression is limited to dorsolateral amygdala. $(E, F)$ Western blot images (picture of the actual immunoblot) and quantifications show an $\sim 50 \%$ decrease in Myosin IIB expression in the LA of animals injected with rAAV expressing a shRNA against Myosin IIB. ${ }^{* *}$ Denotes significantly different from control, $P \leq 0.005$. Error bars represent SEM. 
Indeed, animals that received LatA exhibited significantly impaired LTM compared to animals that received vehicle infusions (Fig. 5B). Upon completion of the experiment, animals were euthanized and histology completed to confirm the location of all infusion needle tips. Infusion needles were found to be well within the lateral amygdala in all animals (Fig. 5C). We then hypothesized that infusion of blebb would mimic the effect of the actin polymerization blocker, LatA. This experiment required four distinct groups (Fig. 6A). Animals first received bilateral intraamygdala infusions of active or inactive blebb, followed $15 \mathrm{~min}$ later by an infusion of either LatA or vehicle. Fifteen min later, animals were fear conditioned and returned to their home cages, and freezing behavior was assessed $24 \mathrm{~h}$ later (Fig. 6B). Consistent with earlier data (Figs. 2B, 5B), animals treated with either blebb or LatA demonstrated significantly impaired long-term fear memory, compared to vehicle-treated controls (Fig. 6C). However, significant levels of freezing (compared to the pretone period) remained in each group (Fig. 6C). Because tone-dependent freezing (freezing levels above pretone freezing) was not completely disrupted, it is possible that mechanisms unrelated to actin polymerization could contribute to cued fear when memories are sufficiently strong. It is also possible that other factors, such as infusion volume or drug concentration, could explain this partial disruption. Interestingly, animals coinfused with both blebb and LatA also displayed reduced levels of freezing compared to vehicle-treated controls (Fig. 6C) but were not significantly more impaired than animals treated with blebb or LatA only (Fig. 6C). In addition, these animals also retained significant tonedependent memory (pretone Blebb + LatA vs. tone Blebb + LatA). Therefore, coinfusion of blebb and LatA together does not result in additive or synergistic disruption to LTM. The most plausible

A

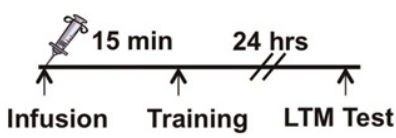

24 Hour LTM Test

B Lat A Infusion $=15$ min pre train



C

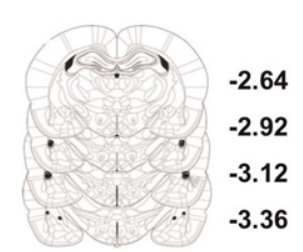

Figure 5. Actin polymerization is required in the lateral amygdala for cued fear memory. $(A)$ Experimental design for intra-LA infusions of LatA. (B) Intra-LA infusions of LatA prior to cued fear conditioning block memory formation $\left(F_{(1,9)}=10.711, P<0.05\right.$; veh, $n=5$; LatA, $n=5$; * denotes significantly different LTM at $24 \mathrm{~h}$ ). (C) Location of needle tips for all intra-LA infusions depicted in $B$.
A

\begin{tabular}{|c|c|c|}
\hline Group & Infusion 1 & Infusion 2 \\
\hline $\mathbf{1}$ & Inactive Blebb & Veh \\
\hline $\mathbf{2}$ & Blebb (Active) & Veh \\
$\mathbf{3}$ & Inactive Blebb & Lat A \\
\hline $\mathbf{4}$ & Blebb (Active) & Lat A \\
\hline
\end{tabular}

B

C



Figure 6. Myosin II motor activity and actin polymerization are involved in a similar molecular process that supports memory consolidation in the lateral amygdala. $(A, B)$ Schematic of experimental design for intra-LA infusions of blebb and LatA. Treatment groups shown in table. (C) Thirtyminute pretraining infusions of blebb and 15 -min pretraining infusions of LatA block LTM (also demonstrated in Figs 2B, 5B), but showed no additive block when infused together $\left(F_{(1,22)}=8.33, P \leq 0.005 ; n=6\right.$, inactive/ veh; $n=6$, blebb/veh; $n=5$, inactive/lat A; $n=6$, blebb/lat A; $P<0.05$ for all groups post hoc comparisons to inactive/veh; ${ }^{*}$ denotes significantly different from vehicle at LTM test). LTM is not completely abolished in any treatment group when compared to pretone freezing $\left(F_{\text {(inactive/veh) }}=\right.$ $689.20, P \leq 0.001 ; F_{(\text {blebb } / \text { veh })}=36.40, P \leq 0.001 ; F_{(\text {inactive } / \text { LatA })}=10.55$, $P \leq 0.01 ; F_{(\text {blebb } / \text { LatA })}=27.28, P \leq 0.001$, denoted by \#). $(D)$ Location of needle tips for all intra-LA infusions. Error bars represent SEM.

explanation for these data is that Myosin II motor activity and actin polymerization are involved in a similar molecular process that subserves memory consolidation. To verify placement of infusion needles, animals were sacrificed following behavioral testing and checked for cannulae placement. All infuser tips were found to be well within the LA in all animals included in behavioral data (Fig. 6D).

\section{Discussion}

In this study, we provide evidence that Myosin II motor activity contributes to memory consolidation in the lateral amygdala. Blocking Myosin II ATPase activity or disrupting expression of Myosin IIB in the LA interfered with LTM formation, which provides strong evidence that Myosin II motor activity is essential for LTM formation. We found that Myosin II motors were specifically required for memory consolidation because disruption of these molecular machines had no effect on learning or memory retrieval. Our finding of memory disruption with a rAAV shRNA targeting Myosin IIB confirmed the results obtained with infusions of a Myosin II ATPase inhibitor. As with blebb, the Myosin IIB-rAAV construct also disrupted memory formation while sparing learning. Thus, Myosin II motor activity and the expression of the specific Myosin II isoform, Myosin IIB, are both required in the LA for fear memories to consolidate. Our present results are similar to data we recently obtained after disruption of Myosin II motors in area CA1 of the hippocampus (Rex et al. 
2010). In that study, both blebb and MyH10 shRNAs disrupted contextual memory formation. STM and memory retrieval were similarly undisturbed after disruption of Myosin II motor activity. Interestingly, LA and CA1 neurons share common molecular processes required for memory formation (Rodrigues et al. 2004). Memories formed in each of these brain areas require NMDAR activation, F-actin polymerization, and AMPAR trafficking to the synapse (Fukazawa et al. 2003; Lamprecht et al. 2006). Synaptic plasticity in both regions exhibits a dependency on shared molecular mechanisms, which closely match the mechanisms required for memory formation (Martin et al. 2000; Maren 2005). We conclude that Myosin II motors participate in a common molecular process supporting memory formation in both the hippocampus and amygdala. This process may be an evolutionarily conserved mechanism subserving information storage in neural circuits throughout the mammalian CNS.

Typically, molecular perturbations that disrupt memory consolidation are also effective when infused after fear conditioning training (Rodrigues et al. 2004). Interestingly, 30 min-post-training infusions of blebb had no effect on memory formation, suggesting that Myosin II motor activity is necessary for memory acquisition. However, preacquisition blebb infusions had no effect on STM, indicating that the cue-shock association was acquired normally and that Myosin II inhibition selectively targeted memory consolidation. This conclusion was confirmed by the lack of effect of blebb on LTM retrieval. We explain this unusual collection of results by proposing that Myosin II-dependent processes that support memory consolidation in the LA are rapidly engaged by learning and are completed within $30 \mathrm{~min}$ of training. We propose that Myosin II motor activity in the LA regulates an early stage of memory consolidation similar to what has been reported for the consolidation of hippocampus-dependent memory (Rex et al. 2010).

In conclusion, our study provides evidence that a Myosin II/ actin-mediated mechanism of consolidation supports fear memories in the lateral amygdala. Myosin II motors are emerging as a promising biological target for the regulation of rapid cognitive processes because of their conserved ability to mobilize actin in neurons. Indeed, Myosin II motor activity is tightly regulated by NMDAR-stimulated signaling pathways known to support memory (Rex et al. 2010). Thus, a detailed understanding of Myosin II mechanics and how this motor contributes to the memory lifecycle is expected to facilitate the discovery of novel approaches to manipulate memory and cognition in neurological and psychiatric disease.

\section{Materials and Methods}

All animal procedures were conducted in accordance with the National Institutes of Health Guide for the Care and Use of Laboratory Animals and with protocols approved by the local Institutional Animal Care and Use Committee.

\section{Animals, cannula implantation, and infusions}

Animals

Adult male Sprague-Dawley rats weighing 275-300 g were used for cued fear conditioning. Rats were housed under 12:12 light/ dark cycles, with food and water available ad libitum.

\section{Cannulae implantation}

For stereotaxic implantation of cannulae, rats were anesthetized with ketamine (90\%) and xylazine (10\%) and secured in a Kopf stereotaxic apparatus. Bilateral stainless steel guide cannulae (26G, Plastics One) were targeted for placement directly above the lateral amygdala (LA) (AP: $-3.0 \mathrm{~mm}$ relative to bregma; ML: $\pm 5.0 \mathrm{~mm}$; DV: $-6.5 \mathrm{~mm}$ from skull) (Paxinos and Watson 1998). Clearance through the guide cannulae was maintained with 33G obdurators (Plastics One) cut to project $1 \mathrm{~mm}$ beyond the tip of the guide. Animals were habituated to dummy cannulae removal and given $5 \mathrm{~d}$ of recovery and handling before the start of any behavioral conditioning.

\section{Infusions}

All infusions were delivered at a rate of $0.25 \mu \mathrm{L} / \mathrm{min}$ for $2 \mathrm{~min}$. Infusers were left in place for $1 \mathrm{~min}$ following the infusion to allow for diffusion of the drug away from needle tips. To ensure accurate cannulae placement, brains were collected after completion of behavioral testing. Cannulated regions of the LA were sliced into $40 \mu \mathrm{m}$ sections and stained with cresyl violet to verify the location of the infusion needle tips. Only those animals with verified placement of infusion needle tips into the LA were included in the statistical analyses of behavioral data.

The concentration of blebb used for in vivo infusions was $90 \mathrm{ng} / \mu \mathrm{L}$ (45 ng of blebb per LA/ per infusion), resulting in a $300 \mu \mathrm{M}$ concentration (Rex et al. 2010). Both active (+) and inactive $(-)$ enantiomers of blebb were first prepared as a stock of $900 \mathrm{ng} / \mu \mathrm{L}$ in $100 \% \mathrm{DMSO}$, then diluted to a final concentration of $90 \mathrm{ng} / \mu \mathrm{L}$ in vehicle consisting of $0.9 \%$ saline and $10 \%$ DMSO (total DMSO $=20 \%$ ). The inactive (+) form of blebb was used as the vehicle for all blebbistatin-related experiments. Latrunculin A (LatA) was used at a concentration of $25 \mathrm{ng} / \mu \mathrm{L}(12.5 \mathrm{ng}$ of LatA per LA). LatA was prepared in a $2 \% \mathrm{DMSO} /$ saline vehicle at a concentration of $60 \mu \mathrm{M}$. Two percent DMSO/saline was used as the vehicle for LatA experiments. LatA and both enantiomers of blebb were purchased from Calbiochem (EMD Chemicals).

\section{Intracranial virus injections}

For intracranial virus injections, rats were anesthetized with ketamine (90\%) and xylazine (10\%) and secured in a Kopf stereotaxic apparatus. A 32-gauge Hamilton syringe was targeted for placement directly below the LA (AP: $-2.8 \mathrm{~mm}$ relative to bregma; ML: $\pm 5.5 \mathrm{~mm}$; DV: $-8.5 \mathrm{~mm}$ from skull) (Paxinos and Watson 1998) and lowered to the target coordinate. Injections were made just below the LA, as virus was found to occupy the needle track and surrounding tissue in pilot studies. Animals were injected with $1 \mu \mathrm{L}$ of recombinant adeno-associated virus (rAAV) expressing GFP and either a scrambled shRNA or a shRNA targeted against the heavy chain of Myosin IIB (Rex et al. 2010). Injections were delivered at a rate of $0.13 \mu \mathrm{L} / \mathrm{min}$.

\section{Behavioral procedure}

\section{Cued fear conditioning}

Auditory cued fear conditioning took place in a Plexiglas chamber that contained a metal grid floor, was lit by a single house light, and was enclosed in a sound attenuating chamber (Med Associates, model ENV-008-FPU). One day prior to training, animals were habituated to the training context for a minimum of 12 total min spread over three exposures. Following conditioning, rats were immediately returned to their home cage. Short-term fear memory was assessed 90 min after training and long-term fear memory was assessed $24 \mathrm{~h}$ post-training.

To validate our paradigm of cued fear conditioning, animals were randomly assigned to paired or unpaired experimental groups. Animals in the unpaired group were subjected to a protocol where the shock (US, $2 \mathrm{sec}, 0.5 \mathrm{~mA}$ ) was administered $1 \mathrm{~min}$ before the tone (CS, $30 \mathrm{sec}$, white noise, $80 \mathrm{db}$ ). Individual sets, but not pairings, of tone-shock exposures were separated by $3 \mathrm{~min}$, with $2 \mathrm{~min}$ of context-only exposure at the beginning of the training trial. Over the course of $12 \mathrm{~min}$ in the chamber, each animal received a total of three tone presentations and three foot shocks, which were delivered such that the tone never coterminated with a foot shock. Paired animals were given 2 min to explore the chamber before being exposed to three pairings of a 30-sec conditioned stimulus (CS, white noise, $80 \mathrm{db}$ ) that 
coterminated with a foot shock unconditioned stimulus (US, 2 sec, $0.5 \mathrm{~mA}$ ). The inter-trial interval varied between 90 and 120 sec. For testing of cued fear memory, the context was made distinct from the training context by changing the walls and floors using Plexiglas inserts, dimming the lights, and adding peppermint oil to scent the chamber. Twenty-four $\mathrm{h}$ after training, animals were exposed to the testing context for $3 \mathrm{~min}$ (novel environment) in the absence of the CS and then exposed to the $\mathrm{CS}$ for $3 \mathrm{~min}$. Freezing behavior was assessed for the entirety of the 3-min CS exposure.

For experiments using blebb, animals received intra-LA infusions of the Myosin II inhibitor or the inactive enantiomer $30 \mathrm{~min}$ prior to conditioning or testing. For experiments that included LatA, animals received infusions of either LatA or appropriate vehicle (2\% DMSO/saline) $15 \mathrm{~min}$ prior to conditioning. When blebb and LatA were infused together, blebb was given $30 \mathrm{~min}$ prior to training, and LatA was given $15 \mathrm{~min}$ prior to training.

\section{Immunoblotting}

Western blotting was performed as previously described (Rex et al. 2010). Briefly, we obtained fresh-frozen tissue punches from the LA of rAAV-injected animals. Tissue was then lysed, samples loaded, and proteins separated by SDS-PAGE. Proteins were then transferred to a PVDF membrane and immunoblotted with antiserum recognizing the Myosin IIB heavy chain (1:100,000, Covance) or $\beta$-tubulin (1:5000, Millipore). The relative expression of Myosin IIB was determined by normalizing the integrated band density values to the values obtained for tubulin on the same blots.

\section{Statistical analysis}

One-way analysis of variance was used to analyze all behavioral data, with one exception. A repeated measures ANOVA and twotailed, paired Student's t-test were used for validation of cued fear conditioning. Immunoblots were evaluated using a two-tailed Student's $t$-test. The Bonferroni post-hoc correction was applied when necessary. Significance was set at $P<0.05$ for all tests.

\section{Acknowledgments}

We thank Jason White, Hope Johnson, and Alicia Brantley for their support and technical assistance. This work was supported by the McKnight Brain Institute, The Scripps Research Institute, and the National Institute for Neurological Disorders and Stroke grant R01-NS064079 (G.R.).

\section{References}

Allingham JS, Smith R, Rayment I. 2005. The structural basis of blebbistatin inhibition and specificity for myosin II. Nat Struct Mol Biol 12: 378-379.

Blair HT, Schafe GE, Bauer EP, Rodrigues SM, LeDoux JE. 2001. Synaptic plasticity in the lateral amygdala: A cellular hypothesis of fear conditioning. Learn Mem 8: 229-242.

Brown ME, Bridgman PC. 2003. Retrograde flow rate is increased in growth cones from myosin IIB knockout mice. J Cell Sci 116: 1087-1094.

Brown ME, Bridgman PC. 2004. Myosin function in nervous and sensory systems. J Neurobiol 58: 118-130.

Dillon C, Goda Y. 2005. The actin cytoskeleton: Integrating form and function at the synapse. Annu Rev Neurosci 28: 25-55.

Dudai Y. 2002. Molecular bases of long-term memories: A question of persistence. Curr Opin Neurobiol 12: 211-216.

Dudai Y. 2004. The neurobiology of consolidations, or, how stable is the engram? Annu Rev Psychol 55: 51-86.

Fischer A, Sananbenesi F, Schrick C, Spiess J, Radulovic J. 2004. Distinct roles of hippocampal de novo protein synthesis and actin rearrangement in extinction of contextual fear. J Neurosci 24: $1962-1966$.

Fukazawa Y, Saitoh Y, Ozawa F, Ohta Y, Mizuno K, Inokuchi K. 2003. Hippocampal LTP is accompanied by enhanced F-actin content within the dendritic spine that is essential for late LTP maintenance in vivo. Neuron 38: $447-460$.

Han JH, Kushner SA, Yiu AP, Hsiang HL, Buch T, Waisman A, Bontempi B, Neve RL, Frankland PW, Josselyn SA. 2009. Selective erasure of a fear memory. Science 323: $1492-1496$.

Hanley JG. 2008. AMPA receptor trafficking pathways and links to dendritic spine morphogenesis. Cell Adh Migr 2: 276-282.

Izquierdo LA, Barros DM, Vianna MR, Coitinho A, deDavid e Silva T, Choi H, Moletta B, Medina JH, Izquierdo I. 2002. Molecular pharmacological dissection of short- and long-term memory. Cell Mol Neurobiol 22: 269-287.

Kovacs M, Toth J, Hetenyi C, Malnasi-Csizmadia A, Sellers JR. 2004. Mechanism of blebbistatin inhibition of Myosin II. J Biol Chem 279: 35557-35563.

Lamprecht R, LeDoux J. 2004. Structural plasticity and memory. Nat Rev Neurosci 5: 45-54

Lamprecht R, Farb CR, Rodrigues SM, LeDoux JE. 2006. Fear conditioning drives profilin into amygdala dendritic spines. Nat Neurosci 9: 481-483.

LeDoux JE. 2000. Emotion circuits in the brain. Annu Rev Neurosci 23: $155-184$.

Limouze J, Straight AF, Mitchison T, Sellers JR. 2004. Specificity of blebbistatin, an inhibitor of myosin II. J Muscle Res Cell Motil 25: 337-341.

Lin CH, Espreafico EM, Mooseker MS, Forscher P. 1996. Myosin drives retrograde F-actin flow in neuronal growth cones. Neuron 16: 769-782.

Malenka RC, Bear MF. 2004. LTP and LTD: An embarrassment of riches. Neuron 44: 5-21.

Mantzur L, Joels G, Lamprecht R. 2009. Actin polymerization in lateral amygdala is essential for fear memory formation. Neurobiol Learn Mem 91: $85-88$.

Maren S. 2001. Neurobiology of Pavlovian fear conditioning. Annu Rev Neurosci 24: 897-931.

Maren S. 2005. Synaptic mechanisms of associative memory in the amygdala. Neuron 47: 783-786.

Martin SJ, Grimwood PD, Morris RG. 2000. Synaptic plasticity and memory: An evaluation of the hypothesis. Annu Rev Neurosci 23: $649-711$.

McGaugh JL. 2000. Memory-a century of consolidation. Science 287: $248-251$.

Medeiros NA, Burnette DT, Forscher P. 2006. Myosin II functions in actinbundle turnover in neuronal growth cones. Nat Cell Biol 8: 215-226.

Morris RG. 2006. Elements of a neurobiological theory of hippocampal function: The role of synaptic plasticity, synaptic tagging, and schemas. Eur J Neurosci 23: 2829-2846.

Paxinos G, Watson C. 1998. The rat brain in stereotaxic coordinates, 4 th ed. Academic Press, London.

Reijmers LG, Perkins BL, Matsuo N, Mayford M. 2007. Localization of a stable neural correlate of associative memory. Science 317: $1230-1233$.

Rex CS, Gavin CF, Rubio MD, Kramar EA, Chen LY, Jia Y, Huganir RL, Muzyczka N, Gall CM, Miller CA, et al. 2010. Myosin IIb regulates actin dynamics during synaptic plasticity and memory formation. Neuron 67: $603-617$.

Rodrigues SM, Schafe GE, LeDoux JE. 2004. Molecular mechanisms underlying emotional learning and memory in the lateral amygdala. Neuron 44: 75-91.

Rogan MT, Staubli UV, LeDoux JE. 1997. Fear conditioning induces associative long-term potentiation in the amygdala. Nature 390: 604-607.

Rubio MD, Johnson R, Miller CA, Huganir RL, Rumbaugh G. 2011. Regulation of synapse structure and function by distinct myosin II motors. J Neurosci 31: 1448-1460.

Ryu J, Liu L, Wong TP, Wu DC, Burette A, Weinberg R, Wang YT, Sheng M. 2006. A critical role for myosin IIb in dendritic spine morphology and synaptic function. Neuron 49: 175-182.

Schafe GE, Nader K, Blair HT, LeDoux JE. 2001. Memory consolidation of Pavlovian fear conditioning: A cellular and molecular perspective. Trends Neurosci 24: 540-546.

Whitlock JR, Heynen AJ, Shuler MG, Bear MF. 2006. Learning induces long-term potentiation in the hippocampus. Science 313: 1093-1097.

Zhou Y, Won J, Karlsson MG, Zhou M, Rogerson T, Balaji J, Neve R, Poirazi P, Silva AJ. 2009. CREB regulates excitability and the allocation of memory to subsets of neurons in the amygdala. Nat Neurosci 12: $1438-1443$.

Received August 29, 2011; accepted in revised form October 25, 2011. 


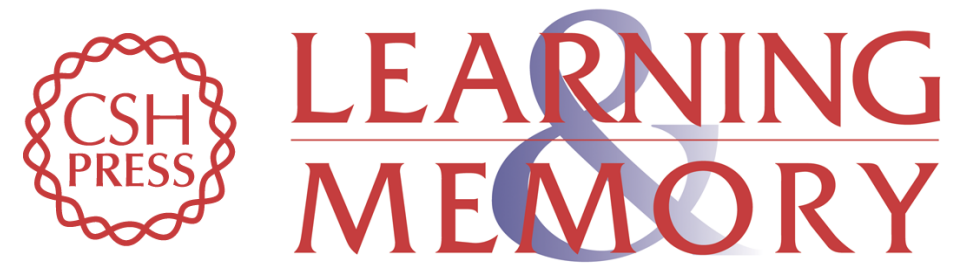

\section{Myosin II motor activity in the lateral amygdala is required for fear memory consolidation}

Cristin F. Gavin, Maria D. Rubio, Erica Young, et al.

Learn. Mem. 2012, 19:

Access the most recent version at doi:10.1101/Im.024042.111

Supplemental http://learnmem.cshlp.org/content/suppl/2011/12/16/19.1.9.DC1
Material

References This article cites 35 articles, 9 of which can be accessed free at: http://learnmem.cshlp.org/content/19/1/9.full.html\#ref-list-1

License

Email Alerting Receive free email alerts when new articles cite this article - sign up in the box at the Service top right corner of the article or click here. 